\title{
Reply to Dr. Rajendram's Letter to Editor: "Point of Care Ultrasound During the Covid-19 Pandemic Should Screen for Inter-Atrial Shunt"
}

\author{
Xi Liu, MD ${ }^{a}$, Faqin Lv, MD ${ }^{b}$, Ji-Bin Liu, MD ${ }^{c, *}$ \\ ${ }^{a}$ Department of Ultrasound, Air Force Medical Center, Air Force Medical University, Beijing, China; ${ }^{b}$ Department of Ultrasound, \\ Hainan Hospital of Chinese PLA General Hospital, Sanya, China; ${ }^{c}$ Editor, AUDT; Department of Radiology, Thomas Jefferson \\ University, Philadelphia, PA, USA
}

Received June 1, 2020; revision received June 25, 2020; accepted June 27, 2020.

Dear Dr. Rajendram and colleagues:

We appreciate your comments on our reviews of the applications of critical care ultrasonography for COVID-19 [1,2]. We have read your opinions with great interest regarding the suggestion and discussion on inter-atrial shunt in COVID-19 patients [3]. The author suggests that COVID-19 patients with four conditions need to be screened for right to left shunt in the heart. This point of view and proposal is a novel potential supplement to various applications of using point of care ultrasound (POCUS) in COVID-19.

Right heart contrast echocardiography (RHCE) is an established technique, which is mainly used to diagnose patent foramen ovale (PFO) and abnormal intrapulmonary circulation [4-7]. It is widely used to screen patients with suspected retro-embolization. There are several commonly-used methods for making a contrast agent suspension for RHCE, including saline plus air ( $9 \mathrm{ml}: 1 \mathrm{ml})$, saline plus air plus autogenous venous blood (9 $\mathrm{ml}: 1 \mathrm{ml}: 1 \mathrm{ml})$, or glucose suspension [8]. In addition, microbubble-based ultrasound contrast agents such as SonoVue (Bracoo, Italy) and Definity (Lantheus, N. Billerica, MA) are commercially available in many countries for contrast-enhanced echocardiography, which could be used with very small dosage for this application if appropriate [9]. In the intensive care unit, contrast ultrasound studies can be conducted at the bedside with portable ultrasound for POCUS examinations.

However, we believe that it is controversial without consensus among the experts for the application of this technique in COVID-19 pandemic situation, especially in patients experiencing a significant decline in pulmonary oxygenation $[1,2,10]$. According to our experiences using right heart contrast echocardiography, we have some concerns and suggestions in term of its limitations for using this method in COVID-19 patients:

(1) The performance of RHCE and the confirming diagnosis of right to left shunt requires the patient to cooperate with Valsalva breathing, which severe patients are unable to do. (2) In actual clinical practice, 2-3 repeated injections are often required to obtain a definitive diagnosis, each time with $10 \mathrm{ml}$ of suspension agent, which will increase the heart and lung load for a critically ill patient. (3) According to the Chinese experts' consensus and guidelines, the patients with cardiopulmonary insufficiency are forbidden to undergo RHCE or should be performed with additional caution [11]. (4) Although the suspension of the contrast agents can be easily made, the scanning procedure and injection timing needs to be coordinated with imaging physicians. If uncoordinated or unpracticed, the syringe and infusion tube may become disconnected, which could increase the risk of infection by infectious blood. (5) Furthermore, even after discovering the PFO or intra-pulmonary shunt by RHCE, patients with mild to moderate shunts may not

\footnotetext{
* Corresponding authors: Editor, AUDT; Department of Radiology, Thomas Jefferson University, 796 Main Building, 132 South 10th Street, Philadelphia, PA 19107, USA

e-mail: ji-bin.liu@jefferson.edu unrestricted use, distribution and reproduction in any medium provided that the original work is properly attributed.
} 
require interventional treatment.

When some patients with large shunts, the feasibility of interventional occlusion of PFO in the severe stages of the COVID-19 pandemic is very limited: First, a highskilled professional team is needed for this treatment. It requires the $\mathrm{C}$-arm $\mathrm{X}$-ray and ultrasound monitoring. The technique involves many people, increasing the probability of infection. Second, because of cytokine storm, COVID-19 patients are prone to develop thrombosis. It is unknown whether the additional procedures will increase the risk of thrombosis, and whether it may be worsened by the contrast examination or the occlusion intervention.

It should be pointed out that the most patients with large shunts could find specific signs and information by using conventional transthoracic echocardiography, including the direct visualization of the "staggered pattern" image of atrial septum, or the thin flap structure in the middle of atrial septum with large "swing". These signs may be helpful for the diagnosis of intracardiac shunt without RHCE.

Therefore, we believe that RHCE could be used in bedside ultrasound, just like for detection of other cardiac structural abnormalities. However, in today's pandemic situation, it is hard to screen COVID-19 patients for PFO shunt. Also, this minimally invasive procedure has to be performed by well-trained professional staff, and it must be evaluated by both echocardiographic experts and clinicians in order to be applied to patients in which it is really necessary. Determining whether occlusion intervention treatment is needed or not also may require a very strict and cautious risk-benefit assessment.

\section{Conflict of Interest}

The authors report no conflict of interest of this research work.

\section{References}

[1] Lv FQ, Wang JR, Yu X, Yang AP, Liu JB, Qian LX, et al. Chinese expert consensus on critical care ultrasound applications at COVID-19 pandemic. Advanced Ultrasound in Diagnosis and Therapy 2020; 4: 27-42.

[2] Liu X, Hai Y, Ma B, Chong W, Liu JB. Critical care ultrasonography and its application for COVID-19. Advanced Ultrasound in Diagnosis and Therapy 2020; 4: 43-49.

[3] Rajendram R, Kharal GA, Mahmood N, Puri R, Kharal M. Rethinking the respiratory paradigm of COVID-19: a 'hole' in the argument. Intensive Care Med 2020:1-2. DOI: 10.1007/s00134-02006102-6.

[4] Homma S, Messé SR, Rundek T, Sun YP, Franke J, Davidson K, et al. Patent foramen ovale. Nat Rev Dis Primers 2016; 2: 15086.

[5] Zhao E, Cheng G, Zhang Y, Li Y, Wang Y. Comparison of different contrast agents in detecting cardiac right-to-left shunt in patients with a patent foramen ovale during contrast-transthoracic echocardiography. Biomed Res Int 2017; 2017: 6086094.

[6] Koh TW. When to use femoral vein injection for diagnosis of patent foramen ovale-Effect of a persistent eustachian valve on right atrial flow patterns during contrast transesophageal echocardiography. Echocardiography 2017; 34: 768-772.

[7] Li X, Gao YH, Wu SZ, Xia HM. Contrast transthoracic echocardiography using $50 \%$ glucose as a contrast agent for screening of a patent foramen ovale. Ultrasound Med Biol 2018; 44 : 2267-2273.

[8] Marriott K, Manins V, Forshaw A, Wright J, Pascoe R. Detection of right-to-left atrial communication using agitated saline contrast imaging: experience with 1162 patients and recommendations for echocardiography. J Am Soc Echocardiogr 2013; 26: 96-102.

[9] Eskandari M, Monaghan M. Contrast echocardiography in daily clinical practice. Herz 2017; 42: 271-278.

[10] Johri AM, Galen B, Kirkpatrick JN, Lanspa M, Mulvagh S, Thamman R. ASE Statement on Point-of-Care Ultrasound during the 2019 Novel Coronavirus Pandemic. J Am Soc Echocardiogr 2020; 33: 670-673.

[11] Chinese Ultrasound Doctors Association. Clinical application guidance of contrast-enhanced echocardiography in China. Beijing: People's Medical Publishing House. 2017. 04; 44. 